\title{
DAS INKULTURATIONSPROBLEM IN HEUTIGER SCHRIFTAUSLEGUNG
}

In der Dogmatischen Konstitution über die göttliche Offenbarung Dei verbum, die vor 20 Jahren auf dem II. Vatikanischen Konzil beschlossen wurde, lesen wir in N. 12: „Weiterhin hat der Erklärer nach dem Sinn $\mathrm{zu}$ forschen, wie ihn aus einer gegebenen Situation heraus der Hagiograph den Bedingungen seiner Zeit und Kultur entsprechend mit Hilfe der damals üblichen literarischen Gattungen - hat ausdrücken wollen und wirklich zum Ausdruck gebracht hat."

Jene ,gegebene Situationen” (determinata adiuncta) betreffen die „Bedingungen der Zeit und Kultur des Hagiographen" (pro sui temporis et suae culturce condicione). Es geht hier also nicht um literaische Gattungen, sondern um jene Arten des Denkens, Sprechens, Erzählens, die zur Zeit des Hagiographen bestanden und aus deren Grunde er diese und keine andere literarische Gattung anwandte. Dass dies tatsächlich so ist, davon zeugen die Aussagen der theologischen Kommission des Konzils vor der Abstimmung über die modi, also die von den Konzilvätern vorgelegten Vorschläge und Änderungen. Sie sind in den Akten des Konzils vermerkt. So wurde beispielsweise auf Antrag eines der Väter die Formel ,seiner Kultur" (suae culturae) eingeführt ${ }^{1}$.

Indem wir von den vorgehend angeführten Daten des Konzildokuments ausgehen, wollen wir uns in diesem Beitrag mit dem Problem befassen, das wir einstweilen als kulturelle Hermeneutik der Bibel oder als Hermeneutik der Inkulturation der Bibel bezeichnen. Um methodisch vorzugehen, teilen wir unsere Erwägungen in vier Teile:

(1) Zuerst geben wir gewisse terminologische Präzisionen an, die uns zur richtigen Darlegung des Problems unerlässlich scheinen.

(2) Weiter verweisen wir an allgemeine hermeneutische Empfehlungen.

(3) Danach führen wir einige Beispiele der Inkulturation von Bibeltexten an, um

(4) Schlussthesen $\mathrm{zu}$ formulieren, die als Forschungsprogramm dienen können.

1 Vgl. Acta Synodalia Sacrosancti Concilii Oecumenici Vaticani II, vol. III, pars III, S. 92 f; vol. IV, pars V, S. 710 f. Dazu: J. Chmiel, Rodzaje literackie $i$ schematy myślenia [Literarische Gattungen und Arten des Denkens]; „Ruch Bibl. i Lit." 38(1985), S. 451 


\section{TERMINOLOGISCHE PRÄZISIONEN}

Es gibt mindestens zwei Termini, die oftmals miteinander identifiziert werden, jedoch unterschiedlichen Inhalts sind. Beide Termini werden öfter promiscue gebracht. Das sind: Akkulturation und Inkulturatio $n^{2}$. Akkulturation - ein der kulturellen Anthropologie entliehener Terminus - bedeutet die Erscheinung eines gegenseitigen Austauschs oder der Osmose zwischen verschiedenen Kulturen. In der englischen Terminologie spricht man von culture contact. Was dies anbetrifft, gibt es überhaupt kein hermeneutisches Problem, da verschiedene Kulturformationen aufeinder stossen und sich gegenseitig durchdringen. Die Erscheinung der Gegenüberstellung von Kulturen tritt seit Anbeginn der Geschichte der Menschheit auf und wird wohl bis zu deren - hoffentlich glücklichen! Ende andauern. Ein echtes hermeneutisches Problem tritt dagegen bei dem zweiten Begriff auf, der Inkulturation, die oft mit der Akkulturation oder auch Enkulturation identifiziert wird. Der Terminus „Inkulturation" ist neuerer Herkunft und bedeutet den Prozess des Eindringens der Offenbarungsbotschaft in die Kultur (bzw. Kulturen), deren Formen, Werte und Sprache ihm die unbedingt notwendigen Mittel zur historischen Veranschaulichung verleihen ${ }^{3}$. Dieser Terminus wurde zum ersten Mal in Kirchendokumenten auf der Bischofssynode im Jahre 1977 angewandt, wo die Rede davon war, dass ,die christliche Botschaft in den menschlichen Kulturen Wurzel fassen (radices agat), sie aufnehmen und umwandeln sollte. Der christliche Glaube sollte sich in die Kulturen einverleiben". Wir haben es hier gewissermassen mit der „Inkarnation des Glaubens" (incarnatio fidei) zu tun. Es ist dies, als würde das evangelische Korn des Glaubens in die Erde der meschlichen Kultur ausgesät, damit diese nach eigener Potenzkraft Früchte trage - wie das in Christi Gleichnis gesagt ist (vgl. Mk 4,8): dreissigfach, ja sechzigfach und hundertfach.

Auf biblischem Grund schreibt Joachim Gnilka von einem solchen Begriff der Inkulturation, obwohl er sich des Terminus Akkulturation bedient ${ }^{4}$. Aber, wie mit recht Alfons Deissler bemerkt, ,da das Christen-

2 Vgl. A.A. Roest-Crollius, What is so new about inculturation? A concept and its implications, "Gregorianum" 59(1978), S. 721-38.

${ }^{3} \mathrm{Vgl}$. Fede e cultura alla luce della Bibbia. Foi et culture à la lumière de la Bible. Actes de la Session plenière 1979 de la Commission Biblique Pontificale, Torino 1981 (= FC); Populus Dei et inculturatio, in: Themata selecta de Ecclesiologia occasione $\mathrm{XX}$ anniversarii conclusionis Concilii Oecumenici Vaticani II (Commissio Theologica Internationalis, Documenta 13), Libreria Ed. Vaticana 1985, S. 24-29; E.J. Korherr, Inkulturation und Katechese in Europa, in: Theologie im Dialog. Fst. zum 400-Jahr-Jubiläum der Kath.-Theol. Fakultät der Universität in Graz, Graz 1985, S. 61-83.

4 Das Akkulturationsproblem nach dem Epheser- und Kolosserbrief; in: FC, S. $235-247$. 
tum nicht einfach mit einer Kultur identifiziert werden kann, scheint [...] das Wort 'Inkulturation' zutreffender zu sein" ${ }^{5}$. Ähnlich benutzt Fritzleo Lentzen-Deis den Terminus intercultural exegesis, als er die Untersuchungsergebnisse unter den Studenten des Pontificio Istituto Biblico in Rom und der Hochschule Sankt Georgen in Frankfurt auf dem Biblischen Kongress in Salamanca im Jahre 1983 referiert ${ }^{6}$. Dem Problem der Inkulturation in der Bibel wurde die Plenarsitzung der Päpstlichen Bibelkommission im Jahre 1979 gewidmet ${ }^{7}$. Es fanden auch zahlreiche Wissenschaftliche Symposien zum Thema der Verhältnisses der Bibeltexte $\mathrm{zu}$ den damaligen Kulturen statt $^{8}$. Dieses Problem ist umso wichtiger und aktueller, als es gewisse gegenwärtige Probleme der Inkulturation des Christentums betrifft, z.B. in die afrikanischen Kulturen ${ }^{9}$. Das Problem der Inkulturation berührt auch Papst Johannes Paul II. in seiner letzten Enzyklika von den hll. Cyryllus und Methodius, den Aposteln der Slaven (Slavorum apostoli), als er von ihrer Einverleibung dieser Kulturen im Leben der Kirche schreibt. Der Papst gebraucht den Terminus „Inkulturation” ${ }^{10}$.

Daraus geht hervor, dass die Inkulturation gegenwärtig eine der wichtigen, wenn nicht sogar der Hauptaufgaben der biblischen Hermeneutik ist. Es ist dies nicht mehr Erklärung des Textes allein, sondern im Zusammenhang mit den Kulturen, in die er aufgenommen wurde und in deren Rahmen er wirkt. Es scheint, dass das Problem erst an Umfang zunimmt und einer positiven Lösung bedarf.

\section{DER KULTURELLE HORIZONT DER HERMENEUTIK}

Gehen wir nun zu einer kurzen Bestimmung des kulturellen Horizonts der Hermeneutik im allgemeinen über. Hans-Georg Gadamer vertrat in seinem monumentalen Werk Wahrheit und Methode ${ }^{11}$ die Ansicht,

5 Das Phänomen von "Dissimilation und Assimilation” im biblischen Offenbarungsgang, dargestellt am Verhältnis von Jahwismus und Baalismus bei Hosea, in: FC, S. $79-92$, hier: S. 79.

${ }^{6}$ Intercultural Exegesis: a Report form a Study Group. (Dem Autor meinen besten Dank für den mir geliehenen Text).

7 FC (vgl. Anm. 1). Dort weitere Literatur.

8 Vgl. dazu: L'uomo nella Bibbia e nelle culture ad essa contemporanee (Atti del Simposio per il XXV dell' A.B.I.), Brescia 1975; Multikulturelles Zusammenleben: theologische Erfahrungen, Frankfurt 1983; J.-P. Thévenaz, Vivons-nous dans une „société multi-culturelle”? Interprétation théologique, „Etudes Théol. et Rel.” $60(1985)$, S. $369-83$.

9 Vgl. L. M. P a sinya, Die Inkulturation der Botschaft des Evangeliums am Beispiel Zaires, "Ordenskorrespondenz" 19(1978), S. 433-44; B. A doukonou, Jalons pour une théologie africaine. Essai d'une herméneutique chrétienne du Vodun dahoméen, vol. I-II, Paris 1984.

10 Slavorum apostoli, N. 21.

11 Wahrheit und Methode. Grundzüge einer philospohischen Hermeneutik, Tübingen ${ }^{41975}(=\mathrm{WM})$. 
der Prozess des Zerfalls des gemeinsamen, in der europäischen Kultur durch griechische und christliche Tradition geschaffenen Horizonts der Interpretation habe im 19. Jh. seinen Anfang genommen. J. Habermas seinerseits nannte die einen Wandel der allumfassenden Interpretation der Welt in subjektive Ethiken und Uberzeugungen ${ }^{12}$. Aufgabe der Hermeneutik ist folglich die Integration der zerstreuten menschlichen Kulturen in eine Folge des Verstehens. Die Grundidee der Interpretation Gadamers ist die Aufgabe des Bewusstseins, die auf dem Erringen derselben eines eigenen hermeneutischen Bewusstseins beruhen soll. Gadamer erachtet die Hermeneutik als Teilnahme an der Kultur. Dies kommt von einer gewissen Horizontverschmelzung. Zitieren wir also Gadamar: „Im Vollzug des Verstehens geschieht eine wirkliche Horizontverschmelzung, die mit dem Entwurf des historischen Horizontes zugleich dessen Aufhebung vollbringt" 13. "Zum wirklichen Verstehen gehört dagegen, die Begriffe einer historischen Vergangenheit so wiederzugewinnen, dass sie zugleich unser eigenes Begreifen mit enthalten" ${ }^{14}$.

Das Verstehen und die Interpretation sollen eigentlich eine normale Lebensaktivität der Menschen sein. „Eine sachangemessene Hermeneutik hätte im Verstehen selbst die Wirklichkeit der Geschichte aufzuweisen" ${ }^{15}$. Gadamer nennt das „Wirkungsgeschichte”. „Verstehen ist seinem Wesen nach ein wirkungsgeschichtlicher Vorgang" ${ }^{16}$. Aufgabe der Hermeneutik ist die Anknüpfung einer neuen semantischen Verbindung mit der Tradition, um auf diese Weise die sprachliche Kommunikation wiederherzustellen. Gadamer stellt fest: „In Wahrheit ist Tradition stets ein Moment der Freiheit und der Geschichte selber" ${ }^{17}$. Wir werden nicht näher auf die philosophische Erwägungen Gadamers eingehen. Nahmen wir daraus nur jene Elemente, die uns erlauben die hermeneutische Aufgabe in der Auslegung der Vergangenheit, also der Tradition zu bestimmen. Wir sind der Annahme, die Kommunikationskonzeption der Kultur, wie Gadamer sie vertritt, fördert unsere These. Drücken wir das deutlicher aus.

In der Welt der Gegenwart erleben wir eine Kulturkrise. Diese Krise hat verschiedene Aspekte, und es ist unmöglich, sie alle hier vorzustellen. Eine Form der Rettung der Kultur ist eine gesunde Kritik der Kultur. Der Philosoph Paul Ricoeur schreibt, dass gegenwärtig ein neuer Zugang zur Wirklichkeit einzig und allein im Schoss der Hermeneutik möglich ist. Nur interpretierend können wir glauben ${ }^{18}$. Deshalb entsteht ein Typ

12 Gekürzt zitiert aus: E. Kobylińska, Hermeneutyczna wizja kultury [Hermeneutic vision of culture], Warszawa-Pıznań 1985, S. 6.

13 WM, S. 290.

14 WM, S. 356.

15 WM, S. 283.

16 Ebd.

17 WM, S. 265.

18 So P. Ricoeur, Symbole donne à penser, „Esprit”, Paris 1959, N. 8. 
von Kultur, die wir eine hermeneutische nennen können, und die Ausdruck des Protestes gegen den konsumptionellen Charakter dieser Zivilisation ist, die die empirische Welt als autarke Qualität anerkannte, und gleichzeitig damit das Stellen definitiver Fragen verbot. Die hermeneutische Kultur ist gewissermassen eine neue „Entzauberung der Welt” ${ }^{19} \mathrm{im}$ Sinne eines Bruchs mit dem Mythus der Allherrschaft der Wissenschaft und der Empirie.

Wir können die Vergangenheit nicht ohne Berücksichtigung unserer Situation erklären. Das Verstehen der Bibel ist keine archäologische Ausgrabung des Sinnes, sondern Verstehen in der Welt, in unserer konkreten Situation, also in unserer Kultur. So wie ein Hagiograph die Gedanken der Offenbarung in den Kategorien seiner Zeit und seiner Kultur ausdrückte, so muss auch der Interpretator sie in den Kategorien seiner Zeit und seiner Kultur heraussuchen. Darauf beruht Gadamers Horizontverschmelzung. Mit anderen Worten, die Auslegung des Sinnes der Bibel erfolgt nicht nur auf historisch-kritischem Wege, obwohl dieser Weg als Anfangsphase für die Rekonstruktion der Vergangenheit unablässlich ist, sondern auf dem Wege der Kommunikation im Sinne der Teilnahme an der Kultur. Daher der Terminus ,hermeneutische Kultur".

Der Bibelinterpretator soll nicht nur den Sinn wiedergeben, also ihn auslegen, sondern eher verstehen. Das Verstehen ist etwas mehr als das Auslegen. Damit ist das Verstehen der Bibeltexte etwas mehr als ihre historisch-literarische Analyse. Es ist die Anknüpfung eines Dialogs mit der Tradition. Es ist die lebendige Sprache des bisher stummen Textes. Lassen wir wiederum Gadamer zu Wort kommen: „Die Stellung zwischen Fremdheit und Vertrautheit, die die Überlieferung für uns hat, ist das Zwischen zwischen der historisch gemeinten Abständlichkeit, Gegenständlichkeit und der Zugehörigkeit zu einer Tradition. In diesem Zwischen ist der wahre Ort der Hermeneutik" ${ }^{20}$. Die hermeneutische Erfahrung des Menschen ist nach Gadamer das Projekt seiner Anteilnahme an der Kultur. Eine vollkommene Teilnahme erfordert das Verstehen der Texte, und vor allem des Wortes Gottes. Auf diese Weise kommen wir zu dem Problem der Sprache, die in der Konzeption Gadamers die Rolle eines gewissen Katalysators der hermeneutischen Erfahrung spielt. Aufgabe der Sprache ist die Horizontverschmelzung, also die Schöpfung jener ewigen Synthese zwischen dem Horizont der Vergangenheit (d.h. dem Text und seiner Tradition) und dem Horizont der Gegenwart (d.h. dem Interpretatoren und seinem Vorverständnis). Die Sprache ist jedoch mit dem Dialog verbunden. Auf diese Weise können und sollen wir durch den Text den

19 Ein Ausdruck von Max Weber.

20 WM, S. 279. 
Dialog beginnen. Dieser Dialog zwischen dem Text und dem Interpretatoren, obwohl er ohne deutliche Grenzen und oft stürmisch verläuft, endet im Augenblick der Auffindung der Wahrheit, die sie in eine neue Form der Vereinigung verbindet. Um mit Bibeltermini zu sprechen, kann und soll die Begegnung mit dem Text zur Metanoia führen, d.h. zur Änderung der Mentalität, zur Vereinigung der Erfahrung mit dem, was hinter dem Text steht ${ }^{21}$.

Dieser kurze Umriss der Kulturvision als symbolische Kommunikation erlaubt uns, zum nächsten Teil unseres Beitrags überzugehen.

MODELLE DER BIBLISCHEN INKULTURATION

Der Inkulturationsprozess am Beispiel ausgewählter Bibeltexte

Greifen wir nun zu einigen ausgewählten Beispielen aus der Bibel, um jenen Inkulturationsprozess gewissermassen aus der Nähe zu zeigen.

1. Wie bereits Henri Cazelles darauf hinweist ${ }^{22}$, ist die Bibel nicht etwa das Buch eines wie in einem Ghetto eingeschlossenen Volkes, sondern von der Genesis bis zur Apokalypse sehen wir das auserwählte Volk mitten in der Geschichte lebend, den nachbarlichen Einflüssen und Einwirkungen ausgesetzt. Noch vor der Berufung Abrahams gibt uns Gen 10 die Völkertafel. Grosse Kulturen beeinflussen Kanaan noch vor der Annexion Josuas: Während die Briefe aus Mari Ägypten nicht erwähnen, bezeugt Ebla den Einfluss ägyptischer Waren auf Syrien, also auch auf Palästinen, bereits im 3. Jahrtausend v. Chr. Eines ist charakteristisch: das auserwählte Volk, das unter David um 1000 v. Chr. zum Staat wird, lässt seine ausserordentliche Vitalität ahnen, indem es schwere Krisen übersteht, denen bedeutende Zivilisation jener Zeit zum Opfer fielen (Ägypten, Babylonien, Anatolien, Ägäa). Die Bibel enthält die bevorrechtigte Erfahrung diese kleinen, von Gott berufenen und von Ihm unter vielen anderen Zivilisationen geführten Volkes. Die Bibel ist nicht mit einer einzigen Kultur verbunden, sie bedient sich vieler Kulturen, um jene einmalige religiöse Erfahrung $\mathrm{zu}$ festigen, die sich in Jesu Christi erfüllt. Lenken wir unsere Aufmerksamkeit auf die Tatsache, dass sich die anfängliche Inkulturation des Wortes Gottes in der sprachlichen Struktur und im bereits literarisch ausgebildeten symbolischen Erbe (Bibelsprachen sowie hebräische, später griechische Schrift) vollzog.

21 Vgl. etwa: V. M a n n u c i, Bibbia come Parola di Dio, Brescia 1981, S. 284; J.-P. Sonnet, La parole consacrée. Théorie des actes de langage, linguistique de l'énonciation parole de la foi, Louvain-la-Neuve 1984.

22 Quelques dettes de l'Ancien Testament envers les cultures ambiantes, in: FC, S. $17-27$. 
2. Vom Gesichtspunkt der Erlösungsgeschichte aus erfolgte der Inkulturationsprozess auf verschiedenen Ebenen. Auf soziopolitischer Ebene musste Israel seine Glauben als zur Extermination verurteiltes Volk verteidigen, als Stämmebündnis, als anfangs mächtiges, später gespaltenes Königsreich, als zerstreutes und einsames Volk unter heidnischen Imperien. Auf kultureller, literarischer bzw. philosophischer Ebene liess Israel bestimmte Einflüsse zu oder es verwarf sie. Während der Ansiedlung in Kanaan machte sich der heidnische Einfluss der Fruchtbarkeitskulte bemerkbar. Seit der Zeit Salomons verspürte man den Einfluss ägyptischer Weiser. In der prämakkabäischen und der makkabäischen Zeit kommt es zum Kontakt mit der hellenistischen Kultur. Üben diese Erfahrungen, die das biblische Volk im Laufe seiner Geschichte machte, nicht die Funktion von Kriterien aus im Inkulturationsprozess, der in Zukunft der Kirche bevorsteht?

3. Im Inkulturationsprozess, der sich im Alten Testament vollzog, begegnen wir einer Erscheinung, die Alfons Deissler als Dissimilation und Assimilation bezeichnete ${ }^{23}$. Worauf beruht sie? Versuchen wir das an zwei Beispieln zu erklären.

Erstes Biespiel: Israel führte unter dem Einfluss des kulturellen Drucks der Nachbarvölker die Monarchie ein als Gewähr der nationalen Unabhängigkeit. Diese Tatsache erweckt Widerstand im Schosse der Prophetentradition als etwas der ausschliesslichen Herrschaft Gottes über Israel Gegensätzliches. Jesaias jedoch entdeckt in der Monarchie eine Vermittlung zwischen Gott und Seinem Volk, sodass die Monarchie zum Modell des Königs Messias wird (messianische Typologie).

Das zweite Beispiel entstammt der Theologie des Propheten Osee. Kanaan ist eine Belohnung Israels, wird aber auch zur Gelegenheit für den Baalismus, also die Kontakte mit dem angetroffenen Heidentum. Osee nennt dies Ehebruch. Die Ehebrecherin Israel jedoch kann sich bekehren und ihr Hochzeitsgeschenk Kanaan wiedererlangen. Es muss also nicht in die Wüste zurück, wie es die Rekabiter wollten.

4. Gehen wir nun zum Neuen Testament über. Jesu bediente sich zum Ausdruck seiner Mission einer symbolischen Sprache zur Bezeichnung der Wirkung und der Existenz des Übels und des Bösen auf der Welt. Jene symbolische Sprache entstand in Anlehnung an das dazumal reiche Material judaistischer Aberglauben. In diesem Sinne schrieb der hl. Paulus: „Als aber die Zeit erfüllt war, sandte Gott seinen Sohn, geboren von einer Frau und dem Gesetz unterstellt" (Gal 4,4). Pierre Grelot schriebt sogar von der Judaizität (Judä̈cité) Jesu ${ }^{24}$. Diese Judaizität ist

23 Vgl. Anm. 3.

24 Jésus devant le „monde du Mal”, in: FC, S. 131-201, hier S. 178. 
nichts anderes als die Summe der kulturellen Erfahrungen Jesu aus dem Bereich des Judaismus. Hätte also doch Bultmann recht mit seinem Postulat der Entmythologisierung? Nein, denn wir dürfen die Sprache nicht ersetzen, in der die Botschaft des Neuen Testaments entstand. Auf dieses Problem kommen wir ganz zum Schluss nachmals zurück.

5. Der Autor des Epheserbriefes bediente sich zur Entwicklung des Begriffs der Universalkirche des hellenistischen Topos von einem, dem lebendigen Organismus ähnlichen Kosmos mit Gliedern und Kopf. In der christlichen Reinterpretation dieser Konzeption handelt es sich nicht mehr um den Kosmos, sondern um die Kirche als Körper und Christus als dessen Kopf $^{25}$.

6. In der Theologie des Johannes ist die Wahrheit (aletheia) Erscheinung Jesu, das bedeutet Wahrheit ist Jesu selbst als Mensch und Gottessohn. Jedoch bereits einige Kirchenväter und Kirchenschriftsteller (Origenes, Augustinus) erliegen der neoplatonischen Philosophie und verleihen dem biblischen Begriff der Wahrheit einen anderen, und zwar ontologischen Sinn. Dies führte später zur Krise der Historizität Jesu ${ }^{26}$.

Im Denken der Gegenwart traten zwei Tendenzen in Erscheinung: Die eine sah die historische Wahrheit in der Vergangenheit. So entstand der übertriebene, hyperkritische Historizismus. Die zweite sah die historische Wahrheit in der Zukunft. Das ist der Weg zum historischen Idealismus Hegels. Der Historizismus lastete fatal auf dem historischen Denken des XIX. Jh.s. Der Idealismus dagegen wurde zum Ausgangspunkt für die Kritik der Geschichte durch Marx: die Wahrheit wurde im politischen Sinne aufgefasst. Die Befreiungstheologie stützt sich heute auf die Orthopraxie und verkündet das Primat des Wirkens, der Praxis vor der Wahrheit.

\section{BEMERKUNGEN IN FORM VON THESEN ALS UMRISS EINES FORSCHUNGSPROGRAMMS}

Versuchen wir nun, eine Art von Forschungsprogramm zu formulieren, das die Gestalt dreier Thesen annimmt. Wir fassen thesenartig einige Bemerkungen zum Inkulturationsproblem in der biblischen Hermeneutik zusammen.

1. Erste These: Der Inkulturationsprozess ist eine in der göttlichen Offenbarung festgestellte Erscheinung.

${ }_{25}$ Vgl. J. Gnilka, Das Akkulturationsproblem, a.a.O. (vgl. Anm. 2), hier S. 236-43. Dazu: H. Köster, Einführung in das Neue Testament im Rahmen der Religionsgeschichte und Kulturgeschichte der hellenistischen und römischen Zeit, Berlin-New York 1980.

${ }_{26}$ Vgl. I. de la Potterie, La notion biblique de vérité et sa rencontre avec la notion hellénistique dans l'Eglise ancienne, in: FC, S. 307-340. 
Dieser Prozess fand von Anfang der Aufzeichnung der Offenbarung an statt. „Da die Heilige Schrift in dem Geist gelesen und ausgelegt werden muss, in dem sie geschrieben wurde" (Dei verbum, 12), sollte die Interpretation der Bibeltexte auch in kulturellen Kontext stattfinden. Tatsächlich, wenn wir die historische Interpretation der Bibel überschauen, müssen wir feststellen, dass die Auslegung der Bibel stets in irgendeinem Kulturkreis erfolgte.

a) Zuerst wurde die Bibel in hellenistischen Kreisen ausgelegt. Hier liegt die grosse Bedeutung der Septuaginta, dank derer die Bibel zum Buch der ganzen (damaligen) Welt wurde.

b) Die zweite Etappe der Bibelinterpretation war der Kulturbereich der Vulgata. Man kann sagen, dass diese Etappe am längsten dauerte, da bis zum II. Vatikanischen Konzil. Die Vulgata war die Grundlage von Übersetzungen, also der ersten Interpretation, in die verschiedenen Nationalsprachen. Beide erwähnte Etappen vollzogen sich auf Grund der Einheit der Interpretation.

c) Die dritte Etappe erleben wir gegenwärtig. Es ist dies die Etappe des Interpretationspluralismus in Anlehnung an die Originaltexte der Bibel, jedoch unter Berücksichtigung der Vielseitigkeit der Ubersetzungen. Sympton dessen kann die Lesung der Heiligen Schrift in verschiedenen Nationalliturgien sein. Jener Pluralismus kann jedoch zu einer gewissen Krise der Interpretation führen, was übrigens zu unserer Zeit deutlich zu beobachten ist.

d) Eine Vision einer neuen Interpretationsetappe ist der Horizont der Jahre 2000, wo eine gewisse Unifizierung in Form der sog. Bibelinformatik zu erwarten ist. Es wird also nicht nur der Text festgelegt (im Original und in Ubersetungen), sondern auch eine entsprechende Anzahl an bereits unifizierten Informationen. Es ist hier weder Zeit noch Platz für eine Beschreibung der daraus entstehenden Gefährdungen. Denken wir lediglich an die möglichen Vorteile.

2. Zweite These: Der Inkulturationsprozess in der Bibelinterpretation verlangt den Bezug auf die Sprache der Offenbarung.

Die Interpretation der Bibel kann die originalen Begriffe nicht ersetzen, die im Prozess der Festigung der Offenbarung eingemeisselt sind. Man kann die Sprache der Bibel - im Sinne von Begriffen - nicht durch die Sprache der einen oder der anderen Philosophie ersetzen. Die Gefahr der „Verphilosophierung” der Bibel durch die Sprache besteht immer. Sehr gut schrieb davon Eugen Biser: „Nicht in einer Übersetzung der biblischen Sprache ins Vokabular der Zeit besteht die Aufgabe, sondern darin, die Sprache der Offenbarung als die wahrhaft zeitgemässe $\mathrm{zu}$ erwisen" ${ }^{27}$. Im Inkulturationsprozess geht es um die Inkarnation des

27 Theologische Sprachtheorie und Hermeneutik, München 1970, S. 568. 
Inhalts (incarnatio fidei) und nicht um Worte. Die Kulturen können sich wandeln, die Grundbegriffe bleiben die gleichen. Jene Kontinuierlichkeit (continuum) der Begriffe garantiert uns die terminologische Schicht biblischer Begriffe.

3. Dritte These: Der Inkulturationsprozess stützt sich auf richtig verstandene Anthropologie ${ }^{28}$.

Es ist dies nämlich ein anthropologischer Prozess, das heisst nicht allein der Begriffe, sondern des Menschen selbst in seinem kulturellen Milieu. Es gibt eine Mannigfaltigkeit der Kulturen, und logischerweise müsste auch eine Verschiedenheit der Interpretation dasein. Richtig sein kann jedoch nur eine einzige Interpretation. Damit aber eine solche einzige - Interpretation die echte ist, muss sie auf einem konstanten Faktor basieren. Ein solcher konstanter Faktor ist der Mensch. Wenn wir von Anthropologie sprechen, denken wir an zwei Ebenen der Anthropologie. Die erste Ebene ist das Phänomen des Menschen: die Existenz der menschlichen Natur ist ein konstanter Faktor. Auf dieser Ebene sind beispielsweise die Gebärden und die Worte Jesu überall und immer was ihren Sinn anbetrifft - verständlich: seine Prophezeiungen, sein Leiden, Kreuz und Auferstehen. Es gibt aber noch eine zweite anthropologische Ebene. Es ist dies die christologische Anthropologie, die darauf beruht, dass wir uns immer als zur Quelle und Ursache der Erscheinung Jesu Christi, Gott und Mensch, unserem Erlöser zuwenden. Das ist der christologische kulturelle Horizont in der Interpretation der Bibel. In dieser Hinsicht sind die Worte der Konstitution Dei verbum (N. 13) belehrend: „Denn Gottes Worte, durch Menschenzunge formuliert, sind menschlicher Rede ähnlich geworden, wie einst des ewigen Vaters Wort durch die Annahme menschlich-schwachen Fleisches den Menschen ähnlich geworden ist".

${ }^{28} \mathrm{Vgl}$. etwa: B. J. M a lin a, The New Testament world. Insights from cultural anthropology, Atlanta 1981; B. L a ng, (Hg.), Anthropological Approche to the Old Testament, Philadelphia-London 1985. 